\title{
Iterative Maximum Likelihood on Networks
}

\author{
Elchanan Mossel ${ }^{*}$ and Omer Tamuz ${ }^{\dagger}$
}

May 4, 2009

\begin{abstract}
We consider $n$ agents located on the vertices of a connected graph. Each agent $v$ receives a signal $X_{v}(0) \sim N(\mu, 1)$ where $\mu$ is an unknown quantity. A natural iterative way of estimating $\mu$ is to perform the following procedure. At iteration $t+1$ let $X_{v}(t+1)$ be the average of $X_{v}(t)$ and of $X_{w}(t)$ among all the neighbors $w$ of $v$. It is well known that this procedure converges to $X(\infty)=\frac{1}{2}|E|^{-1} \sum d_{v} X_{v}$ where $d_{v}$ is the degree of $v$.

In this paper we consider a variant of simple iterative averaging, which models "greedy" behavior of the agents. At iteration $t$, each agent $v$ declares the value of its estimator $X_{v}(t)$ to all of its neighbors. Then, it updates $X_{v}(t+1)$ by taking the maximum likelihood (or minimum variance) estimator of $\mu$, given $X_{v}(t)$ and $X_{w}(t)$ for all neighbors $w$ of $v$, and the structure of the graph.

We give an explicit efficient procedure for calculating $X_{v}(t)$, study the convergence of the process as $t \rightarrow \infty$ and show that if the limit exists then $X_{v}(\infty)=X_{w}(\infty)$ for all $v$ and $w$. For graphs that are symmetric under actions of transitive groups, we show that the process is efficient. Finally, we show that the greedy process is in some cases more efficient than simple averaging, while in other cases the converse is true, so that, in this model, "greed" of the individual agents may or may not have an adverse affect on the outcome.

The model discussed here may be viewed as the Maximum-Likelihood version of models studied in Bayesian Economics. The ML variant is more accessible and allows in particular to show the significance of symmetry in the efficiency of estimators using networks of agents.
\end{abstract}

\footnotetext{
${ }^{*}$ Weizmann Institute and U.C. Berkeley. E-mail: mossel@stat.berkeley.edu. Supported by a Sloan fellowship in Mathematics, by BSF grant 2004105, by NSF Career Award (DMS 054829) by ONR award N00014-07-1-0506 and by ISF grant $1300 / 08$

${ }^{\dagger} 9$ Smilanski St., Herzlia 46361, Israel
} 


\section{Introduction}

Networks and graphs are often viewed as computational models. In computational complexity several complexity classes are studied in terms of corresponding computation graphs, for example finite-automata, PSPACE and LOG-SPACE. For general background see, e.g., [1]. In parallel computing, networks are used to model the communication network between different computers, while in sparse sensing the connectivity network is of fundamental computation significance (see, e.g., [2] and [9]).

A recent trend emanating from Economics and Game Theory considers networks where different nodes correspond to computational entities with different objectives [5]. Recent models in Bayesian Economics consider models where each player is repeatedly taking actions that are based on a signal he has received that is correlated with the state of the word and past actions of his neighbors ([8], [3], 7]).

In this paper we study a simple model where, in each iteration, agents iteratively try to optimally estimate the state of the world, which is a single parameter $\mu \in \mathbb{R}$. It is assumed that originally each agent receives an independent sample from a normal distribution with mean $\mu$. Later at each iteration each agent updates his estimate by taking the maximum likelihood estimator of $\mu$ given its current estimator and those of its neighbors, and given the graph structure. Note that for normal distributions, the maximum likelihood estimator is identical with the minimum variance unbiased estimator. At the first iteration, the estimator at each node will be the average of the original signal at the node and its neighbors. However, from the second iteration on, the procedure will not proceed by simple averaging due to the correlation between the estimators at adjacent nodes. As we show below, this correlation can be calculated given the structure of the graph and results in dramatic differences from the simple averaging process. Note that under this model, the agents are memoryless and use only the results of the last iteration to calculate those the next.

The model suggested above raises a few basic questions:

- Is the process above well defined?

- Can the estimators be efficiently calculated? Note that in the Bayesian economic models (such as [7]) there are no efficient algorithms for updating beliefs.

- Does the process converge?

We answer the first two questions positively, and conjecture that the answer to the third is positive as well. Once these questions are addressed we prove a number of results regarding the limit estimators including:

- We show that for connected graphs, as $t \rightarrow \infty$, the correlation between the estimators of the different agents goes to one.

- We describe a graph for which the maximum likelihood process converges to an estimator different than the optimal.

- We compare the statistical efficiency of the limiting estimator to the limiting estimator obtained by simple iterative averaging and to the optimal estimator, in different graphs.

\subsection{Formal Definition of the Model}

We consider a finite, undirected, connected graph $G=(V, E)$, where each vertex has a self-loop so that $\forall v:(v, v) \in E$, and a state of the world $\mu \in \mathbb{R}$. We assign each vertex $v$ a normal unbiased 
estimator $X_{v}=X_{v}(0)$ of $\mu$ so that $\mathbf{E}\left[X_{v}\right]=\mu$ and $\operatorname{Var}\left[X_{v}\right]=1$, for all $v$. These estimators are uncorrelated.

In iteration $t \in \mathbb{N}$ we define $X_{v}(t+1)$ to be the minimum variance unbiased estimator constructible over the estimators of $v$ and its neighbors $N(v)=\{w \mid(v, w) \in E\}$ at time $t$

$$
\begin{gathered}
X_{v}(t+1)=\sum_{w \in N(v)} \alpha_{w} X_{w}(t), \quad \text { where: } \\
\sum_{w \in N(v)} \alpha_{w}=1, \text { and } \alpha \text { minimizes } \operatorname{Var}\left[\sum_{w} \alpha_{w} X_{w}(t)\right] .
\end{gathered}
$$

(note that $\alpha$ may be positive or negative). The process $Y_{v}$ is given by simple iterative averaging so $Y_{v}(0):=X_{v}$, and

$$
Y_{v}(t+1)=\frac{1}{\mathrm{~d}_{\mathrm{v}}} \sum_{w \in N(v)} Y_{w}(t) .
$$

It is well known that $Y_{v}(t)$ converges to $Y_{v}(\infty)=\frac{1}{2}|E|^{-1} \sum d_{v} X_{v}$.

Finally, we define $Z(\infty)$ to be the global minimum variance unbiased estimator:

$$
Z(\infty)=\frac{1}{|V|} \sum_{v \in V} X_{v}
$$

Note that in the case of normally distributed $X_{v}$ 's, the minimum variance definitions coincide with those of maximum likelihood.

This scheme can be generalized to the case where the original estimators $X_{v}$ have a general covariance structure, with the definitions for $X_{v}(t)$ and $Y_{v}(t)$ remaining essentially the same, and that of $Z(\infty)$ changing to the form of Eq. 4 below.

\subsection{Statements of the main results}

- The process defined by Eqs. 1 and 2 is well defined. More formally:

Proposition (2.1). For every realization of the random variables $X_{v}(0), v \in V$ and for all $t \geq 1, X_{v}(t)$ is uniquely determined.

- The process can be calculated efficiently:

Proposition (2.2). It is possible to calculate $\left\{X_{v}(t) \mid v \in V\right\}$, given $\left\{X_{v}(t-1) \mid v \in V\right\}$, by performing $n$ operations of finding the point of an $n$ dimensional affine space (as specified by a generating set of size at most $n$ ) with minimal $L_{2}$ norm.

Calculating the latter is a classical convex optimization problem. See, e.g., [4].

- For transitive graphs the process always converges to the optimal estimator:

Proposition (2.7). Let $G$ be a transitive graph (defined below). Then $X_{v}(t)$ converges to $X(\infty)=Z(\infty)$.

- For graphs of large maximal degree, $X_{v}(t)$ converge to $\mu$ :

Proposition (3.1). Let $G_{n}=\left(V_{n}, E_{n}\right)$ be a family of graphs where $\left|V_{n}\right|=n$ and $\max _{v \in V_{n}} d_{v} \rightarrow$ $\infty$. Then

$$
\lim _{n \rightarrow \infty} \sup _{v \in V_{n}} \lim _{t \rightarrow \infty} \mathbf{E}\left[\left(X_{v}(t)-\mu\right)^{2}\right]=0 .
$$


Note by comparison that for any graph,

$$
\mathbf{E}\left[(Y(\infty)-\mu)^{2}\right]=\frac{1}{4}|E|^{-2} \sum_{v \in V} d_{v}^{2} .
$$

In particular for a star on $n$ vertices, as $n \rightarrow \infty$ it holds that $X(\infty)$ converges to $\mu$ but $Y(\infty)$ does not.

- Finally, for some graphs, the process converges to a limit different than $Z(\infty)$ and $Y(\infty)$.

Theorem (A.1). Let $G=(V, E)$ be the interval of length four where $V=\{a, b, c, d\}$ and $E=\{\{a, b\},\{b, c\},\{c, d\}\}$. Then $X_{v}(t)$ converges to a limit $X(\infty)$, where

$$
X(\infty)=\frac{1}{4}\left[(1-\xi)\left(X_{a}+X_{d}\right)+(1+\xi)\left(X_{b}+X_{c}\right)\right], \quad \operatorname{Var}[X(\infty)]=\xi,
$$

with $\xi=2-\sqrt{3}=\frac{1}{4}(1+\sqrt{49}-\sqrt{48})$.

Note that for this graph

$$
Y(\infty)=\frac{1}{4}\left(\left(1-\frac{1}{5}\right)\left(X_{a}+X_{d}\right)+\left(1+\frac{1}{5}\right)\left(X_{b}+X_{c}\right)\right), \quad \operatorname{Var}[Y(\infty)]=0.26,
$$

and

$$
Z(\infty)=\frac{1}{4}\left(X_{a}+X_{b}+X_{c}+X_{d}\right), \quad \operatorname{Var}[Z(\infty)]=\frac{1}{4}
$$

\subsubsection{Conjectures}

Showing some supporting results, we conjecture that the process always converges, and in particular to a state where all agents have the same estimator.

We present a number of additional open problems and conjectures in the conclusion.

\section{General Proofs}

\subsection{Process is Well Defined}

Proposition 2.1. For every realization of the random variables $X_{v}(0), v \in V$ and for all $t \geq 1$, $X_{v}(t)$ is uniquely determined.

Proof. Let $X_{v}^{(1)}(t)=A$ and $X_{v}^{(2)}(t)=B$ be minimum variance estimators satisfying Eqs. 1 and 2, with variance $V$. Then their average must have variance at least $V$, since it also is a linear combination of the estimators from which $A$ and $B$ were constructed:

$$
\begin{aligned}
V & \leq \operatorname{Var}\left[\frac{1}{2}(A+B)\right] \\
V & \leq \frac{1}{4} \operatorname{Var} A+\frac{1}{4} \operatorname{Var} B+\frac{1}{2} \operatorname{Cov}(A, B) \\
V & \leq \frac{1}{2} V+\frac{1}{2} \operatorname{Cov}(A, B) \\
V & \leq \operatorname{Cov}(A, B) .
\end{aligned}
$$

Since $\operatorname{Cov}(A, B) \leq \sqrt{\operatorname{Var} A \operatorname{Var} B}=V$, then $\operatorname{Cov}(A, B)=V$ and $A=B$. Therefore, there exists a unique minimum variance unbiased estimator and the process is well defined. 


\subsection{The Algorithm for Calculating the Estimator}

We present an efficient algorithm to calculate $X_{v}(t)$. Let $E_{v}(t)=\left\{X_{w}(t) \mid w \in N(v)\right\}$ be the estimators of agent $v$ 's neighbors at time $t$. Let $\mathbf{C}$ be the covariance matrix of $E_{v}(t)$, so that $C_{w u}=\operatorname{Cov}\left(X_{w}(t), X_{u}(t)\right)$. For each $w$, let $x_{w}$ be a realization of $X_{w}(t)$. Then the log likelihood of $y \in \mathbb{R}$ is

$$
\log \mathcal{L}(y)=-\sum_{w u}\left(x_{w}-y\right) C_{w u}^{-1}\left(x_{u}-y\right)+\text { const },
$$

where $\mathbf{C}^{-1}$ is $\mathbf{C}$ 's pseudo-inverse. this expression is maximal for

$$
y=\frac{\sum_{w u} C_{w u}^{-1} x_{w}}{\sum_{w u} C_{w u}^{-1}} .
$$

Hence, the MLE, and therefore also $X_{w}(t+1)$, equals

$$
X_{M L}=X_{v}(t+1)=\frac{\sum_{w, u \in N(v)} C_{w u}^{-1} X_{w}(t)}{\sum_{w, u \in N(v)} C_{w u}^{-1}} .
$$

Note that $X_{v}(t+1)$ is also, among all the unbiased estimators of $\mu$ constructible over the estimators in $E_{V}(t)$, the one with the minimum variance.

Given this last observation, there exists a simple geometric interpretation for Eq. 4:

Proposition 2.2. It is possible to calculate $\left\{X_{v}(t) \mid v \in V\right\}$, given $\left\{X_{v}(t-1) \mid v \in V\right\}$, by performing $n$ operations of finding the point of an $n$ dimensional affine space (as specified by a generating set of size at most $n$ ) with minimal $L_{2}$ norm.

Proof. Consider an $n$-dimensional vector space $\mathcal{V}$ over $\mathbb{R}$, with an inner product $\langle\cdot, \cdot\rangle$. Let $\mathbf{z}$ be some non-zero vector in $\mathcal{V}$, and let $\mathcal{A} \subset \mathcal{V}$ be the affine space defined by $\mathcal{A}=\{\mathbf{x} \in \mathcal{V} \mid\langle\mathbf{x}, \mathbf{z}\rangle=1\}$.

(This is a generalization of $\mathcal{V}=\operatorname{span}\left(\left\{X_{v} \mid v \in V\right\}\right),\langle X, Y\rangle=\operatorname{Cov}(X, Y), \mathbf{z}=\sum_{v \in V} X_{v}$ and $\mathcal{A}$ being the set of unbiased estimators).

Given a set of vectors $E=\left\{\mathbf{x}_{k} \mid k=1, \ldots, K \leq n\right\}$, where $\mathbf{x}_{k} \in \mathcal{A}$, let $C_{k l}=\left\langle\mathbf{x}_{k}, \mathbf{x}_{l}\right\rangle$. Then $\mathcal{A} \cap \operatorname{span}(E)$ is also an affine space, and the minimum $L_{2}$ norm vector in $\mathcal{A} \cap \operatorname{span}(E)$ is

$$
\mathbf{x}_{M L}=\frac{\sum_{k l} C_{k l}^{-1} \mathbf{x}_{k}}{\sum_{k l} C_{k l}^{-1}}
$$

where $\mathbf{C}^{-1}$ is the matrix pseudo-inverse of $\mathbf{C}$. This equation is identical to Eq. 4 .

Note that if $\mathbf{C}$ is invertible then its pseudo-inverse is equal to its inverse. Otherwise, there are many linear combinations of the vectors in $E$ which are equal to the unique $\mathbf{x}_{M L}$. The rôle of the pseudo-inverse is to facilitate computation: it provides the linear combination with least sum of squares of the coefficients [6].

\subsection{Convergence}

Denote $V_{v}(t):=\operatorname{Var}\left[X_{v}(t)\right]$ and $C_{v w}(t):=\operatorname{Cov}\left(X_{v}(t), X_{w}(t)\right)$.

Lemma 2.3. All estimators have the same limiting variance: $\exists \rho_{\infty} \forall v: V_{v}(t) \rightarrow \rho_{\infty}$ 
Proof. Since, in every iteration, each agent calculates the minimum variance unbiased estimator over those of his neighbors and its own, then the variance of the estimator it calculates must be lower than that of any other unbiased linear combination:

$$
\sum_{w \in N(v)} \alpha_{w}=1 \Rightarrow V_{v}(t+1) \leq \operatorname{Var}\left[\sum_{w \in N(v)} \alpha_{w} X_{w}(t)\right] .
$$

In particular, for each neighbor $w$ of $v$

$$
V_{v}(t+1) \leq V_{w}(t)
$$

and since each vertex is its own neighbor, then

$$
V_{v}(t+1) \leq V_{v}(t)
$$

Therefore, since the variance of each agent's estimator is monotonously decreasing (and positive), it must converge to some $\rho_{v}$. Now assume $(v, w) \in E$ and $\rho_{v}<\rho_{w}$, then, at some iteration $t$, $V_{v}(t)<\rho_{w} \leq V_{w}(t)$. But then, by Eq. 6, we have $V_{w}(t+1) \leq V_{v}(t)<\rho_{w}$ - a contradiction. Therefore, $\rho_{v}$ must equal $\rho_{w}$, and since the graph is connected, all agents must converge to the same variance, $\rho_{\infty}$.

Lemma 2.4. $\forall v, w: C_{v w}(t) \rightarrow \rho_{\infty}$

Proof. The previous lemma is a special case of this one, for when $v=w$. Otherwise, for two neighboring agents $v$ and $w$, for any $\epsilon$, there exists an iteration $t$ where both $\operatorname{Var}\left[X_{v}(t)\right]<\rho_{\infty}+\epsilon$ and $\operatorname{Var}\left[X_{w}(t)\right]<\rho_{\infty}+\epsilon$. Then:

$$
\operatorname{Var}\left[\frac{1}{2}\left(X_{v}(t)+X_{w}(t)\right)\right]=\frac{1}{4}\left[V_{v}(t)+V_{w}(t)+2 C_{v w}(t)\right]<\frac{1}{2}\left[\rho_{\infty}+\epsilon+C_{v w}(t)\right]
$$

and since Eq. 5 implies $V_{v}(t+1) \leq \operatorname{Var}\left[\frac{1}{2}\left(X_{v}(t)+X_{w}(t)\right)\right]$, then $\rho_{\infty}<\frac{1}{2}\left[\rho_{\infty}+\epsilon+C_{v w}(t)\right]$ and $C_{v w}(t) \geq \rho_{\infty}-\epsilon$. Since $C_{v w}$ is also bounded from above: $C_{v w}(t) \leq \sqrt{\operatorname{Var}\left[X_{v}(t)\right] \operatorname{Var}\left[X_{w}(t)\right]}<$ $\rho_{\infty}+\epsilon$, we have demonstrated that $C_{v w}(t) \rightarrow \rho_{\infty}$ when $v$ and $w$ are neighbors. This implies that the correlation between neighbors converges to 1 , and therefore, since the graph is finite, all correlations converge to 1 and all covariances converge to $\rho_{\infty}$.

This last lemma implies that if one agent's estimator converges, then all others' also converge, to the same limit. Even without convergence, however, it implies that all the estimators converge to their average:

$$
\lim _{t \rightarrow \infty} \operatorname{Var}\left[X_{v}(t)-\frac{1}{|V|} \sum_{w \in V} X_{w}(t)\right]=0 .
$$

The following lemma will be used to conjecture that all the estimators do converge. It states that an estimator is uncorrelated to the difference between it and any of the estimators which were used to calculate it.

Lemma 2.5. $\forall w \in N(v): \operatorname{Cov}\left(X_{v}(t+1), X_{v}(t+1)-X_{w}(t)\right)=0$

Proof. We examine the estimators $\hat{X}(\beta)=X_{v}(t+1)(1-\beta)+X_{w}(t) \beta$, which are also unbiased estimators of $\mu$, and are linear combinations of the estimators from which $X_{v}(t+1)$ was constructed. They should all therefore have higher variance than $X_{v}(t+1)$. Since $\hat{X}(\beta=0)=X_{v}(t+1)$, then

$$
0=\left.\frac{\partial \operatorname{Var}[\hat{X}]}{\partial \beta}\right|_{\beta=0} .
$$


Now:

$$
\begin{aligned}
0 & =\left.\frac{\partial \operatorname{Var}[\hat{X}]}{\partial \beta}\right|_{\beta=0} \\
& =\left.\frac{\partial\left[(1-\beta)^{2} \operatorname{Var}\left[X_{v}(t+1)\right]+\beta^{2} \operatorname{Var}\left[X_{w}(t)\right]+2 \beta(1-\beta) \operatorname{Cov}\left(X_{v}(t+1), X_{w}(t)\right)\right]}{\partial \beta}\right|_{\beta=0} \\
& =\left.\left[-2(1-\beta) \operatorname{Var}\left[X_{v}(t+1)\right]+2 \beta \operatorname{Var}\left[X_{w}(t)\right]+2(1-2 \beta) \operatorname{Cov}\left(X_{v}(t+1), X_{w}(t)\right)\right]\right|_{\beta=0} \\
& =-2 \operatorname{Var}\left[X_{v}(t+1)\right]+2 \operatorname{Cov}\left(X_{v}(t+1), X_{w}(t)\right) \\
& =-2 \operatorname{Cov}\left(X_{v}(t+1), X_{v}(t+1)-X_{w}(t)\right)
\end{aligned}
$$

and so $\operatorname{Cov}\left(X_{v}(t+1), X_{v}(t+1)-X_{w}(t)\right)=0$.

Note that this implies that $\operatorname{Var}\left[X_{v}(t+1)\right]=\operatorname{Cov}\left(X_{v}(t+1), X_{w}(t)\right)$.

Conjecture 2.6. (Convergence) $\exists X(\infty) \forall v: X_{v}(t) \rightarrow X(\infty)$.

The following observation supports this conjecture:

$$
\begin{aligned}
\operatorname{Var}\left[X_{v}(t+1)-X_{v}(t)\right] & =\operatorname{Cov}\left(X_{v}(t+1)-X_{v}(t), X_{v}(t+1)-X_{v}(t)\right) \\
& =\operatorname{Cov}\left(X_{v}(t+1), X_{v}(t+1)-X_{v}(t)\right)-\operatorname{Cov}\left(X_{v}(t), X_{v}(t+1)-X_{v}(t)\right)
\end{aligned}
$$

Using Lemma 2.5

$$
\begin{aligned}
& =-\operatorname{Cov}\left(X_{v}(t), X_{v}(t+1)-X_{v}(t)\right) \\
& =V_{v}(t)-\operatorname{Cov}\left(X_{v}(t+1), X_{v}(t)\right)
\end{aligned}
$$

and using it again:

$$
=V_{v}(t)-V_{v}(t+1) .
$$

This implies that if $t_{0}$ is such that $V_{v}\left(t_{0}\right)=\rho_{\infty}+\epsilon$ and therefore $\sum_{t=t_{0}}^{\infty} V_{v}(t)-V_{v}(t+1)=\epsilon$, then

$$
\sum_{t=t_{0}}^{\infty} \operatorname{Var}\left[X_{v}(t+1)-X_{v}(t)\right]=\epsilon .
$$

\subsection{Efficiency for Transitive Graphs}

Vertex transitive graphs (henceforth referred to as transitive graphs), are graphs where all vertices are essentially equivalent, or "equally important". Alternatively, one may say that the graph "looks the same" from all vertices. Formally, $G=(V, E)$ is transitive iff, for every pair of vertices $v, w \in V$ there exists a function $f: V \rightarrow V$ which is a graph automorphism (i.e. $f$ is a bijection and $(a, b) \in E \Leftrightarrow(f(a), f(b)) \in E)$ and maps $v$ to $w$.

Proposition 2.7. When $G$ is transitive then the process converges and $X(\infty)=Z(\infty)$.

Proof. By the symmetry of the graph, the average of the agents' estimators cannot give more weight to one agent's original estimator than to another:

$$
\frac{1}{|V|} \sum_{v} X_{v}(t)=\frac{1}{|V|} \sum_{v} X_{v}=Z(\infty),
$$


and hence the average of the agents' estimators is constant and in particular converges. By lemma 2.4 (Eq. 8), if the average converges then each of the estimators converges to the same limit:

$$
\forall v \lim _{t \rightarrow \infty} X_{v}(t)=\lim _{t \rightarrow \infty} \frac{1}{|V|} \sum_{v} X_{v}(t)=Z(\infty)=X(\infty) .
$$

Note that for regular graphs (i.e. graphs where all vertices have the same degree), which are a superset of transitive graphs, $Y(\infty)=Z(\infty)$.

\section{Analytic Examples}

Complete analytical analysis of these iterations for general graphs seems difficult, since Eq. 4 is quadratic. In fact, we found only two simple examples amenable to complete analysis: The star, a graph with a central node connected to all other nodes, and the interval of length four, a graph of four linearly ordered nodes.

In the former, we show that the minimum variance scheme is efficient, so that $X(\infty)=Z(\infty)$. In the latter, we show that it isn't, but that the "price of anarchy" is low.

\subsection{High degree graphs and the star}

We consider a graph of $n$ vertices, of which $u$ is the central node and is connected to all others, and no additional edges exist.

The averaging estimator $Y(\infty)$ gives weight $\frac{n}{3 n-2}$ to $X_{u}$ and $\frac{2}{3 n-2}$ to the rest. Its variance is $\frac{n^{2}+4 n-4}{(3 n-2)^{2}}$, which is asymptotically $\frac{1}{9}$.

On the other hand, $X_{u}(1)=Z(\infty)$, since node $u$, neighboring all nodes of the graph, immediately finds the global minimum variance estimator. In the next iteration, all nodes $w$ set $X_{w}(2)=X_{u}(1)$, and the process essentially halts, since all nodes have the same estimator, $X_{w}(2)=$ $X(\infty)=Z(\infty)$, with $\operatorname{Var}[X(\infty)]=\frac{1}{n}$.

In general, in graphs of large maximal degree, $X_{v}(t)$ converges to $\mu$ :

Proposition 3.1. Let $G_{n}=\left(V_{n}, E_{n}\right)$ be a family of graphs where $\left|V_{n}\right|=n$ and $\max _{v \in V_{n}} d_{v} \rightarrow \infty$. Then

$$
\lim _{n \rightarrow \infty} \sup _{v \in V_{n}} \lim _{t \rightarrow \infty} \mathbf{E}\left[\left(X_{v}(t)-\mu\right)^{2}\right]=0 .
$$

Proof. Since all estimators at all iterations have mean $\mu$, then $\mathbf{E}\left[\left(X_{v}(t)-\mu\right)^{2}\right]=\operatorname{Var}\left[X_{v}(t)\right]$. By lemma 2.3 , the limiting variances of all the agents in a graph $G_{n}$ are equal to some $\rho_{n}$, and therefore

$$
\lim _{n \rightarrow \infty} \sup _{v \in V_{n}} \lim _{t \rightarrow \infty} \mathbf{E}\left[\left(X_{v}(t)-\mu\right)^{2}\right]=0 \quad \leftrightarrow \quad \lim _{n \rightarrow \infty} \rho_{n}=0
$$

The condition $\max _{v \in V_{n}} d_{v} \rightarrow \infty$ implies that given $\epsilon>0$, there exists a high enough $N$, so that in any $G_{n}$ with $n>N$ there exists a node $w_{n}$ with degree $d_{w_{n}}$ larger than $1 / \epsilon$. Then $\operatorname{Var}\left[X_{w_{n}}(1)\right]<\epsilon$, since agent $w_{n}$ would, on the first iteration, average the estimators of all its neighbors, resulting in a new estimator of variance $1 / d_{w_{n}}$. Since variance never increases in the iterative process (lemma 2.3), then $\rho_{n}<\epsilon$ for $n$ larger then some $N$. Since this is true for arbitrary $\epsilon, \rho_{n}$ goes to zero as $n$ goes to infinity. 


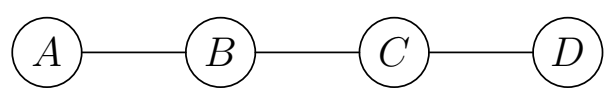

Figure 1: Interval of Length Four

\subsection{Interval of Length four}

We analyze the case of $G=(V, E)$ where $V=\{a, b, c, d\}$ and $E=\{\{a, b\},\{b, c\},\{c, d\}\}$.

In Appendix $\mathrm{A}$, we prove that the process converges with

$$
\operatorname{Var}[X(\infty)]=2-\sqrt{3}=\frac{1}{4}(1+\sqrt{49}-\sqrt{48})>\frac{1}{4}=\operatorname{Var}[Z(\infty)],
$$

thus proving that a case exists where $X(\infty) \neq Z(\infty)$. We also, for this case, derive an asymptotic convergence rate of $2-\sqrt{3}$.

The averaging estimator $Y(\infty)$ is:

$$
Y(\infty)=0.2 X_{a}+0.3 X_{b}+0.3 X_{c}+0.2 X_{d}
$$

with

$$
\operatorname{Var}[Y(\infty)]=0.26
$$

This is slightly lower than $\operatorname{Var}[X(\infty)]$, which equals about 0.268 . However, the convergence rate (second eigenvalue) for the averaging process is $\frac{1}{4}+\sqrt{33} / 12 \approx 0.73$, which is significantly slower than the minimum variance process's asymptotic rate of $2-\sqrt{3} \approx 0.268$.

\section{$4 \quad$ Numerical Examples and Conjectures}

Numerical simulations on intervals of lengths larger than four suggest a surprising result.

\subsection{Interval of Arbitrary Length}

Numerical simulations suggest that $X(\infty)$, for intervals of length $n$, approaches a normal distribution around the center of the interval, with variance proportional to $n$ :

Conjecture 4.1. For interval graphs of length $2 n$, index the agents by $k \in\{0, \ldots, 2 n-1\}$. Then

$$
X(\infty)=\sum_{k} A_{k} X_{k}
$$

where $A_{k}$ approaches a normal distribution in the sense that

$$
\lim _{n \rightarrow \infty} \sum_{k}\left(A_{k}-C_{n} e^{-(k-n+1 / 2)^{2} / \nu(n)}\right)^{2}=0 \quad \text { with } \quad \nu(n) \in \Theta(n), C_{n} \in \mathbb{R} .
$$

This implies that while $\lim _{n \rightarrow \infty} \mathbf{E}\left[(X(\infty)-\mu)^{2}\right]=0, X(\infty)$ quickly becomes less efficient when compared to $Z(\infty)$ :

Conjecture 4.2. $\operatorname{Var}[X(\infty)] \propto \sqrt{n} \operatorname{Var}[Z(\infty)]$

Note that $\operatorname{Var}[Y(\infty)]$, on the other hand, approaches $\operatorname{Var}[Z(\infty)]$ as $n$ increases, for intervals of length $n$. 


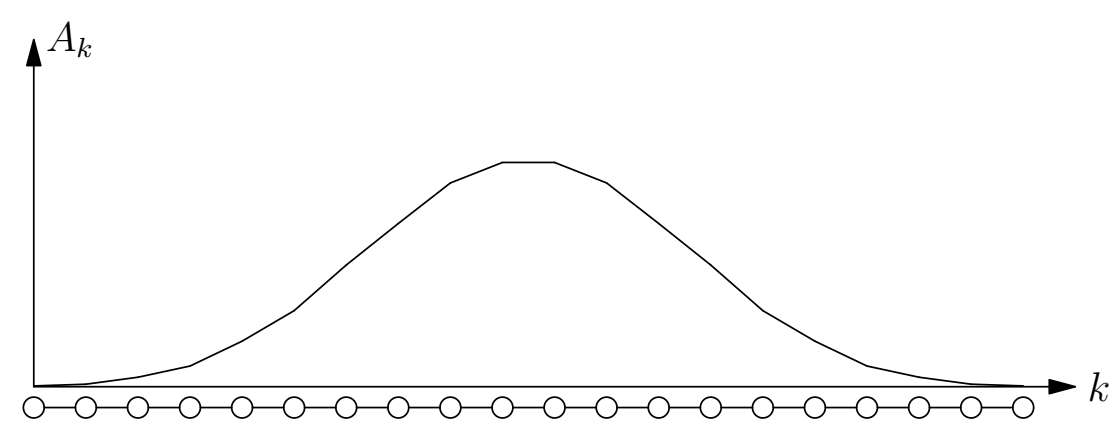

Figure 2: Simulation of interval of length 20.

\subsection{Agents with Memory}

A model which is perhaps more natural than the memoryless model is the model in which the agents remember all their own values from the previous iterations.

Proposition 4.3. If the agents have memory, then the process converges to $Z(\infty)$.

Proof. Since each vertex $v$ always remembers $X_{v}=X_{v}(0)$, then $X_{v}$ is always part of the set over which $X_{v}(t)$ was constructed. Then, by Lemma 2.5 :

$$
\operatorname{Cov}\left(X_{v}(t), X_{v}\right)=\operatorname{Var}\left[X_{v}(t)\right]
$$

and by Lemma 2.4;

$$
\forall v, w \in V: \lim _{t \rightarrow \infty} \operatorname{Cov}\left(X_{w}(t), X_{v}\right)=\lim _{t \rightarrow \infty} \operatorname{Cov}\left(X_{v}(t), X_{v}\right)=\lim _{t \rightarrow \infty} \operatorname{Var}\left[X_{v}(t)\right]=\rho_{\infty} .
$$

This means that for any agent $w$, the covariance of its limit estimator with each of the original estimators $X_{v}$ is identical, and so it must be their average: $X(\infty)=Z(\infty)$.

This proof relied only on the agents' memory of their original estimators. Since they also gain more estimators over the iterations, and seemingly expand the space that they span, we conjecture that:

Conjecture 4.4. $\forall v \in V: X_{v}(t)=X(\infty)$, for $t \geq|V|$.

\section{Conclusion}

An number of interesting open problems can be raised with respect to this model, some of which we conjecture about above:

- Does it always converge? We conjecture above that this is indeed the case.

- For what graphs does it converge to the optimal estimator $Z(\infty)$ ?

- Otherwise, what is the "price of anarchy", $\operatorname{Var}[X(\infty)] / \operatorname{Var}[Z(\infty)]$ ? Is it bounded? We conjecture above that it isn't.

- What is the convergence rate? 


\section{A Analysis of Interval of Length Four}

Theorem A.1. Let $G=(V, E)$ be the interval of length four where $V=\{a, b, c, d\}$ and $E=$ $\{\{a, b\},\{b, c\},\{c, d\}\}$. Then $X_{v}(t)$ converges to a limit $X(\infty)$, where

$$
X(\infty)=\frac{1}{4}\left[(1-\xi)\left(X_{a}+X_{d}\right)+(1+\xi)\left(X_{b}+X_{c}\right)\right], \quad \operatorname{Var}[X(\infty)]=\xi,
$$

with $\xi=2-\sqrt{3}=\frac{1}{4}(1+\sqrt{49}-\sqrt{48})$.

Proof. We define $M_{v w}(t)=\operatorname{Cov}\left(X_{v}, X_{w}(t)\right)$, so that each column of $\mathbf{M}$ is the coordinates of an agent's estimator at time $t$, viewed as a vector in the space spanned by $\left\{X_{a}, X_{b}, X_{c}, X_{d}\right\}$. We define $Z(\infty)$-subtracted $M$ as $\tilde{M}_{v w}(t)=\operatorname{Cov}\left(X_{v}-Z(\infty), X_{w}(t)-Z(\infty)\right)$, where $Z(\infty)=$ $\frac{1}{4}\left(X_{a}+X_{b}+X_{c}+X_{d}\right)$, and likewise define the $Z(\infty)$-subtracted covariance matrix $\tilde{C}_{v w}(t)=$ $\operatorname{Cov}\left(X_{v}(t)-Z(\infty), X_{w}(t)-Z(\infty)\right)$.

We now shift to an alternative orthonormal basis $B$ :

$$
B=\left\{\left(\begin{array}{c}
1 / 2 \\
1 / 2 \\
1 / 2 \\
1 / 2
\end{array}\right)\left(=b_{1}\right),\left(\begin{array}{c}
-1 / \sqrt{2} \\
0 \\
0 \\
1 / \sqrt{2}
\end{array}\right)\left(=b_{2}\right),\left(\begin{array}{c}
0 \\
-1 / \sqrt{2} \\
1 / \sqrt{2} \\
0
\end{array}\right)\left(=b_{3}\right),\left(\begin{array}{c}
-1 / 2 \\
1 / 2 \\
1 / 2 \\
-1 / 2
\end{array}\right)\left(=b_{4}\right)\right\}
$$

The vector $b_{1}(=2 Z(\infty))$ was chosen because its coordinate is one half in every unbiased estimator (and zero for any $Z(\infty)$-subtracted unbiased estimator). $b_{2}$ and $b_{3}$ are anti-symmetric to inversion of the interval, a transformation which should leave $X(\infty)$ invariant by the symmetry of the graph. Therefore we expect their coordinates in $X(\infty)$ to vanish. We have no freedom, then, in choosing the last vector, and expect $X(\infty)$ to equal $\frac{1}{2} b_{1}$ plus some constant $\xi$ times $\frac{1}{2} b_{4}$ :

$$
X(\infty)=\frac{1}{4}\left[(1-\xi)\left(X_{a}+X_{d}\right)+(1+\xi)\left(X_{b}+X_{c}\right)\right]
$$

Performing two iterations of the process reveals that under this basis, the $Z(\infty)$-subtracted coordinates matrix of the estimators at iteration two, $\tilde{\mathbf{M}}_{V}(2)$, is of the form:

$$
\tilde{\mathbf{M}}_{B}(2)=\left(\begin{array}{cccc}
0 & 0 & 0 & 0 \\
x & 0 & 0 & -x \\
0 & z & -z & 0 \\
y & w & w & y
\end{array}\right)
$$

with

$$
y w=z^{2}+w^{2}
$$

Application of another iteration yields a matrix of the same form:

$$
\tilde{\mathbf{M}}_{B}(3)=\left(\begin{array}{cccc}
0 & 0 & 0 & 0 \\
0 & \frac{-x z}{x^{2}+(y-w)^{2}} z & \frac{x z}{x^{2}+(y-w)^{2}} z & 0 \\
z & 0 & 0 & -z \\
w & \frac{x^{2}}{x^{2}+(y-w)^{2}} w & \frac{x^{2}}{x^{2}+(y-w)^{2}} w & w
\end{array}\right)
$$

with the relation of Eq. 10 preserved.

Since the result is a matrix of essentially the same form, equivalent equations apply for consecutive iterations, and we may denote as $x_{t}, y_{t}, w_{t}$ and $z_{t}$ the corresponding matrix entries at time $t$. 
Since Eq. 11 implies that $y_{t}=w_{t-1}$ and $x_{t}=z_{t-1}$, then if $w_{t}$ and $z_{t}$ converge then the process converges and $X(\infty)$ exists. Also:

$$
w_{t+1}=\frac{z_{t-1}^{2}}{z_{t-1}^{2}+\left(w_{t-1}-w_{t}\right)^{2}} w_{t}
$$

and

$$
z_{t+1}=\frac{z_{t-1} z_{t}}{z_{t-1}^{2}+\left(w_{t-1}-w_{t}\right)^{2}} z_{t}
$$

Dividing Eq. 12 by Eq. 13, we discover that:

$$
\frac{w_{t+1}}{z_{t+1}}=\frac{z_{t-1}}{z_{t}} \frac{w_{t}}{z_{t}}
$$

and therefore, by repeated application:

$$
w_{t}=\frac{w_{2} z_{2}}{z_{3}} \frac{z_{t}}{z_{t-1}}=\frac{1}{2} \frac{z_{t}}{z_{t-1}}
$$

Eq. 10 can alternatively be written as: $w_{t-1} w_{t}=w_{t}^{2}+z_{t}^{2}$. Then:

$$
w_{t-1}-w_{t}=z_{t}^{2} / w_{t}=2 z_{t} z_{t-1},
$$

and we can write Eq. 13 as:

or

$$
z_{t+1}=\frac{z_{t-1} z_{t}}{z_{t-1}^{2}+4 z_{t}^{2} z_{t-1}^{2}} z_{t}
$$

$$
\frac{z_{t}}{z_{t+1}}=\frac{z_{t-1}}{z_{t}}\left(1+4 z_{t}^{2}\right)
$$

To solve this recursion we make the following guess:

$$
\frac{z_{t}}{z_{t+1}}=2+\sqrt{3-4 z_{t}^{2}}
$$

which is a solution of the following quadratic equation in $z_{t} / z_{t+1}$ :

$$
\frac{z_{t}^{2}}{z_{t+1}^{2}}-4 \frac{z_{t}}{z_{t+1}}+1+4 z_{t}^{2}=0
$$

This is equivalent to the following relation:

$$
\operatorname{Var}\left[X_{b}(t)\right]=w_{t}^{2}+z_{t}^{2}+\frac{1}{4}=2 w_{t},
$$

upon which we serendipitously stumbled during our examination of this problem.

This guess satisfies Eq. 14, as some manipulation of the two equations will show. Since $z_{2}$ and $z_{3}$ satisfy Eq. 15, then the rest of the $z$ 's must, too.

Since Eq. 14 implies $z_{t} \rightarrow 0$, we can conclude from $\frac{z_{t-1}}{z_{t}}=2+\sqrt{3-4 z_{t-1}^{2}}$ that

$$
\lim _{t \rightarrow \infty} \frac{z_{t+1}}{z_{t}}=2-\sqrt{3}:=\xi
$$

This is the process's asymptotic convergence rate. Since $w_{t}=\frac{1}{2} \frac{z_{t}}{z_{t-1}}$, then $w_{t} \rightarrow \frac{1}{2} \xi$, and

$$
X(\infty)=\frac{1}{4}\left[(1-\xi)\left(X_{a}+X_{d}\right)+(1+\xi)\left(X_{b}+X_{c}\right)\right]
$$

with

$$
\operatorname{Var}[X(\infty)]=\left(\frac{1}{2}\right)^{2}+\left(\frac{1}{2} \xi\right)^{2}=\xi
$$




\section{References}

[1] S. Aaronson, G. Kuperberg, and C. Granade. The complexity zoo. At: qwiki.stanford.edu/wiki/Complexity_Zoo.

[2] H. M. Ammari and S. K. Das. Integrated coverage and connectivity in wireless sensor networks: A two-dimensional percolation problem. IEEE Trans. Computers, 57(10):1423-1434, 2008.

[3] A. V. Banerjee. A simple model of herd behavior. The Quarterly Journal of Economics, 107(3):797-817, 1992.

[4] S. Boyd and L. Vandenberghe. Convex Optimization. Cambridge University Press, 2004.

[5] M. Jackson. The economics of social networks. In R. Blundell, W. Newey, and T. Persson, editors, Volume I of Advances in Economics and Econometrics, Theory and Applications: Ninth World Congress of the Econometric Society, pages 1-56. Cambridge University Press, 2006.

[6] W. Press, S. Teukolsky, W. Vetterling, and B. Flannery. Numerical recipes in C. Cambridge University Press, 1992.

[7] L. Smith and P. Sorensen. Pathological outcomes of observational learning. Econometrica, 68(2):371-398, 2000.

[8] D. H. Sushil Bikhchandani and I. Welch. Learning from the behavior of others: Conformity, fads, and informational cascades. The Journal of Economic Perspectives, 12(3):151-170, 2008.

[9] H. Zhang and J. C. Hou. Asymptotic critical total power for k-connectivity of wireless networks. IEEE/ACM Trans. Netw., 16(2):347-358, 2008. 